\title{
Palifermin for Oral Mucositis after Intensive Therapy for Hematologic Cancers
}

\author{
Ricardo Spielberger, M.D., Patrick Stiff, M.D., William Bensinger, M.D., \\ Teresa Gentile, M.D., Ph.D., Daniel Weisdorf, M.D., Tarun Kewalramani, M.D., \\ Thomas Shea, M.D., Saul Yanovich, M.D., Keith Hansen, M.D., \\ Stephen Noga, M.D., Ph.D., John McCarty, M.D., C. Frederick LeMaistre, M.D., \\ Eric C. Sung, D.D.S., Bruce R. Blazar, M.D., Dieter Elhardt, Ph.D., \\ Mon-Gy Chen, M.S., and Christos Emmanouilides, M.D.
}

From the City of Hope National Medica Center, Duarte, Calif. (R.S.); Loyola University Medical Center, Maywood, III. (P.S.) the Fred Hutchinson Cancer Research Center, Seattle (W.B.); State University of New York Upstate Medical University, Syracuse (T.G.); the University of Minnesota, Minneapolis (D.W., B.R.B.); Memorial SloanKettering Cancer Center, New York (T.K.) the University of North Carolina, Chape Hill (T.S.); Georgetown University Cance Center, Washington, D.C. (S.Y.); the Northwest Marrow Transplant Center, Portland Oreg. (K.H.); Sinai Hospital of Baltimore, Baltimore (S.N.); the Medical College of Virginia, Richmond (J.M.); the Texas Transplant Institute, San Antonio (C.F.L.); the School of Dentistry (E.C.S.) and the Division of Hematology/Oncology (C.E.), University of California at Los Angeles, Los Angeles; and Amgen, Thousand Oaks, Calif. (D.E., M.-G.C.). Address reprint requests to Dr. Spielberger at the City of Hope National Medical Center, Department of $\mathrm{He}$ matology and Hematopoietic Cell Trans plantation and Kaiser Permanente BMT Program, 1500 E. Duarte Rd., Duarte, CA 91010, or at rspielberger@coh.org.

N Engl J Med 2004;351:2590-8.

Copyright $@ 2004$ Massachusetts Medical Society.
ABSTRACT

\section{BACK GROU N D}

Oral mucositis is a complication of intensive chemotherapy and radiotherapy with no effective treatment. We tested the ability of palifermin (recombinant human keratinocyte growth factor) to decrease oral mucosal injury induced by cytotoxic therapy.

\section{METHODS}

This double-blind study compared the effect of palifermin with that of a placebo on the development of oral mucositis in 212 patients with hematologic cancers; 106 patients received palifermin ( $60 \mu \mathrm{g}$ per kilogram of body weight per day) and 106 received a placebo intravenously for three consecutive days immediately before the initiation of conditioning therapy (fractionated total-body irradiation plus high-dose chemotherapy) and after autologous hematopoietic stem-cell transplantation. Oral mucositis was evaluated daily for 28 days after transplantation.

\section{RESULTS}

The incidence of oral mucositis of World Health Organization (WHO) grade 3 or 4 was 63 percent in the palifermin group and 98 percent in the placebo group $(\mathrm{P}<0.001)$. Among patients with this degree of mucositis, the median duration of mucositis was 6 days (range, 1 to 22 ) in the palifermin group and 9 days (range, 1 to 27 ) in the placebo group. Among all patients, regardless of the occurrence of mucositis, the median duration of oral mucositis of WHO grade 3 or 4 was 3 days (range, 0 to 22) in the palifermin group and 9 days (range, 0 to 27 ) in the placebo group ( $\mathrm{P}<0.001)$. As compared with placebo, palifermin was associated with significant reductions in the incidence of grade 4 oral mucositis ( 20 percent vs. 62 percent, $\mathrm{P}<0.001$ ), patient-reported soreness of the mouth and throat (area-under-the-curve score, 29.0 [range, 0 to 98] vs. 46.8 [range, 0 to 110]; $\mathrm{P}<0.001$ ), the use of opioid analgesics (median, $212 \mathrm{mg}$ of morphine equivalents [range, 0 to 9418 ] vs. $535 \mathrm{mg}$ of morphine equivalents [range, 0 to 9418], $\mathrm{P}<0.001$ ), and the incidence of use of total parenteral nutrition (31 percent vs. 55 percent, $\mathrm{P}<0.001$ ). Adverse events, mainly rash, pruritus, erythema, mouth and tongue disorders, and taste alteration, were mild to moderate in severity and were transient.

CONCLUSIONS

Palifermin reduced the duration and severity of oral mucositis after intensive chemotherapy and radiotherapy for hematologic cancers. 
$\mathrm{H}$ IGH-DOSE CHEMOTHERAPY AND RAdiotherapy followed by hematopoietic stem-cell support is a well-established treatment for hematologic cancers. Oral mucositis develops and requires treatment in approximately 70 to 80 percent of patients receiving radiationbased conditioning treatments. ${ }^{1,2}$ The incidence and severity of oral mucositis vary with the conditioning regimen.

Oral mucositis results from injury to epithelial cells that line the oral cavity. The damage causes changes ranging from mild atrophy to severe ulceration. Serious consequences include pain requiring opioid analgesia, potentially life-threatening infections, inadequate nutrition requiring parenteral feeding, and prolonged hospitalization. ${ }^{1-5}$ Currently, no standard therapy prevents or treats severe oral mucositis. ${ }^{6,7}$

Keratinocyte growth factor is a $28-\mathrm{kD}$, heparinbinding member of the family of fibroblast growth factors that was originally isolated from pulmonary fibroblasts as a protein (FGF-7) with keratinocytestimulating activity. Palifermin (recombinant human keratinocyte growth factor) is an $\mathrm{N}$-terminal, truncated version of endogenous keratinocyte growth factor with biologic activity similar to that of the native protein, but with increased stability. ${ }^{8}$ In animal models of chemotherapy, radiotherapy, and hematopoietic stem-cell transplantation, ${ }^{9,10}$ palifermin protected several types of epithelial tissues. A phase 1 trial indicated that palifermin at doses of up to $80 \mu \mathrm{g}$ per kilogram of body weight per day for three consecutive days was not associated with major adverse events. ${ }^{11}$ We evaluated the efficacy and safety of palifermin in reducing the duration and severity of oral mucositis and its sequelae (soreness of the mouth and throat, use of opioid analgesics and total parenteral nutrition, and infections) in patients with hematologic cancers who were undergoing autologous hematopoietic stemcell transplantation after receiving total-body irradiation and high-dose chemotherapy.

\section{METHODS}

\section{PATIENTS}

The institutional review board at each of the 13 study sites approved the study protocol; all patients gave written informed consent before all study-related procedures. This study enrolled patients who were at least 18 years of age, had a Karnofsky performance-status score of at least 70 , and were sched- uled to undergo autologous stem-cell transplantation after receiving a conditioning regimen ${ }^{12-15}$ of fractionated total-body irradiation plus etoposide and cyclophosphamide for non-Hodgkin's lymphoma, Hodgkin's disease, acute myelogenous leukemia, acute lymphoblastic leukemia, chronic lymphocytic leukemia, or multiple myeloma. Patients were to have at least $1.5 \times 10^{6}$ cryopreserved CD34+ cells per kilogram available for transplantation and adequate cardiac, pulmonary, renal, and hepatic function, as determined by institutional guidelines.

\section{STUDY DRUG}

Palifermin and its matching placebo were manufactured and packaged by Amgen. The placebo contained all the ingredients of the palifermin preparation (i.e., $10 \mathrm{mM}$ histidine [pH 6.5], 2 percent sucrose, 4 percent mannitol, and 0.01 percent polysorbate 20) except palifermin.

\section{STUDY DESIGN}

In this placebo-controlled, double-blind, phase 3 trial, patients were randomly assigned in a 1:1 ratio (stratified according to center and type of hematologic cancer) to receive palifermin $(60 \mu \mathrm{g}$ per kilogram per day) or placebo intravenously for three consecutive days, starting three days before the initiation of total-body irradiation. After the conditioning regimen was administered, patients received three additional doses ${ }^{16}$ of palifermin or placebo on days 0,1 , and 2 after transplantation. Filgrastim (Neupogen, Amgen) (5 $\mu$ g per kilogram per day) was administered to all patients daily from the day of transplantation until the neutrophil count recovered.

Conditioning therapy and supportive care were administered according to standard institutional practice. Total-body irradiation (total, 1200 cGy) was delivered in 6,8 , or 10 fractions over a period of three or four days, with at least six hours between fractions, before chemotherapy was administered. Chemotherapy included intravenous etoposide (60 mg per kilogram) the day after the last radiation fraction (four days before transplantation) and one dose of cyclophosphamide (100 mg per kilogram) two days before transplantation. Patients received peripheral-blood hematopoietic stem cells collected after mobilization by means of either cytokines or chemotherapy with or (for one patient) without cytokines.

Oral mucositis was assessed with the use of three scales: the five-grade World Health Organization 
(WHO) oral-toxicity scale ${ }^{17}$ (primary scale), the fivegrade Radiation Therapy Oncology Group (RTOG) acute radiation-morbidity scoring criteria for mucous membranes, ${ }^{18}$ and the four-grade Western Consortium for Cancer Nursing Research (WCCNR) revised staging system for oral mucositis. ${ }^{19}$ At each center, Clinical Assistance Programs trained evaluation-team members to assess and document oral mucositis. Each patient was assessed daily for oral mucositis beginning 8 days before transplantation and continuing for 28 days after transplantation or until severe oral mucositis had resolved (i.e., returned to WHO grade 0,1 , or 2 ).

Data on patient-reported outcomes (soreness of the mouth and throat and ability to participate in the activities of daily living) were collected with the use of a daily questionnaire. The functional and physical well-being domains of the Functional Assessment of Cancer Therapy general questionnaire ${ }^{20}$ were collected daily from 12 days before transplantation to 28 days after transplantation (total, 41 days).

Information concerning the use of parenteral or

\begin{tabular}{|lcc|}
\hline \hline Table 1. Baseline Characteristics of the Patients. & \\
\hline & $\begin{array}{c}\text { Palifermin } \\
\text { Group } \\
\text { (N=106) }\end{array}$ & $\begin{array}{c}\text { Placebo } \\
\text { Group } \\
\text { (N=106) }\end{array}$ \\
Characteristic & $59(56)$ & $72(68)$ \\
Male sex - no. (\%) & & \\
Age - yr & 48 & 49 \\
Median & $18-69$ & $19-68$ \\
Range & & \\
Diagnosis - no. (\%) & $72(68)$ & $69(65)$ \\
Non-Hodgkin's lymphoma & $21(20)$ & $23(22)$ \\
Hodgkin's disease & $11(10)$ & $9(8)$ \\
Multiple myeloma & $2(2)$ & $5(5)$ \\
Leukemia* & & \\
Karnofsky performance-status score - no. (\%) & $3(3)$ & $1(1)$ \\
70 & $15(14)$ & $19(18)$ \\
80 & $59(56)$ & $58(55)$ \\
90 & $29(27)$ & $28(26)$ \\
100 & & \\
Total no. of CD34+ cells reinfused - $\times 10^{-6} / \mathrm{kg}$ & 5.2 & 5.0 \\
Median & $1.8-87.0$ & $1.5-41.0$ \\
Range & & \\
\hline
\end{tabular}

* This category includes acute lymphoblastic leukemia (one patient in the palifermin group), acute myelogenous leukemia (one patient in the palifermin group and four in the placebo group), and chronic lymphocytic leukemia (one patient in the placebo group) in complete remission. transdermal narcotic analgesics was collected daily. Total parenteral nutrition was given according to the standards of each study site.

Antibodies against palifermin were assayed by an electrochemiluminescence-based immunoassay (IGEN International). Any positive test was followed by retesting for neutralizing antibodies with a cell-based assay. Laboratory assessments included routine hematologic and blood chemical tests and measurement of serum amylase and lipase concentrations.

The study was designed by Amgen and the principal investigators, who also recruited the patients and collected the data. The investigators and Ms. Chen and Dr. Elhardt of Amgen analyzed the data; all investigators had access to the data. The decision to publish was made by the investigators and Amgen; no limitations were imposed by the sponsor. The lead investigator wrote the article with editorial assistance from Amgen.

\section{EFFICACY AND SAFETY END POINTS}

Analyses of all efficacy and safety end points included all patients who underwent randomization and received at least one dose of study medication. The prespecified primary end point was the duration of oral mucositis of WHO grade 3 (inability to swallow solid food) or 4 (no form of oral alimentation possible); the duration was considered to be zero days among patients who did not have oral mucositis.

Other end points included the incidence of oral mucositis of WHO grade 3 or 4 , the duration of oral mucositis of WHO grade 3 or 4 among patients with this adverse effect, the incidence of WHO grade 4 oral mucositis, the duration of WHO grade 4 oral mucositis among patients with this adverse effect, the duration of oral mucositis of WHO grade 2 (moderate) or higher, the duration of oral mucositis of RTOG grade 3 or 4 , the duration of lesions of WCCNR grade 2 or 3, the area under the curve (AUC) for patient-reported outcomes of soreness of the mouth and throat and swallowing limitations plotted against time, scores for the Functional Assessment of Cancer Therapy general questionnaire, and the total dose and duration of parenteral or transdermal opioid analgesics. Additional exploratory end points included the incidence of febrile neutropenia, the incidence of infections, and the incidence of the use of total parenteral nutrition.

Safety was assessed on the basis of the incidence of adverse events, graded on a five-point scale 
(a score of 1 indicates mild adverse effects, and a score of 5 fatal adverse effects); changes in clinical laboratory values; the occurrence of antibodies against palifermin; and the rates of progression-free survival and secondary cancers during follow-up.

\section{STATISTICAL ANALYSIS}

The planned sample size of 210 patients (105 per group) was chosen to provide the study with the statistical power to detect a minimal mean $( \pm S D)$ difference between groups in the duration of severe oral mucositis of more than $3.0 \pm 6.6$ days, with the use of a t-test with 90 percent power and a two-sided significance level of 5 percent. For the end points involving the duration of oral mucositis, missing data with two adjacent observed assessments were assigned the poorer of the two adjacent values. For each patient whose oral mucositis had not resolved by the end of the study we imputed the duration by assigning the mean duration among all patients who had severe oral mucositis for as long as or longer than the patient had it before the study ended. Treatment groups were compared by means of the generalized Cochran-Mantel-Haenszel method stratified according to study center. ${ }^{21}$ The test statistic was based on the within-stratum standardized midranks. Secondary end points were evaluated in a prespecified order only after the primary end point was determined to be statistically significant. All statistical tests were two-sided at the 0.05 level of significance. The Kaplan-Meier method ${ }^{22}$ was used to estimate the rate of progression-free survival.

\section{RESULTS}

\section{PATIENTS}

Between March 23, 2001, and October 23, 2002, 245 patients were screened, 214 (107 per group) were randomly assigned to a treatment group, and 212 (106 per group) received at least one dose of palifermin or placebo and were included in the safety and efficacy analyses. The center that enrolled the most patients enrolled 19 percent of them.

The baseline characteristics of the patients were similar in the two groups (Table 1). Of the $212 \mathrm{pa}-$ tients, 88 (83 percent) in the palifermin group and 86 (81 percent) in the placebo group had a baseline Karnofsky performance-status score of at least 90. All patients had received prior chemotherapy; 88 percent of those in the palifermin group and 92 percent of those in the placebo group had received no prior radiotherapy. Radiotherapy schedules were similar in the two groups. A combination of chemotherapy and cytokines was used for CD34+ cell mobilization in 75 percent of patients in the palifermin group and 72 percent of patients in the placebo group. Most patients ( 92 percent of those in the palifermin group and 93 percent of those in the placebo group) received acyclovir or a similar antiviral drug. Similar numbers of patients in both groups completed the study: 103 patients in the palifermin

\begin{tabular}{|c|c|c|c|}
\hline Variable & $\begin{array}{l}\text { Palifermin } \\
\text { Group } \\
(\mathrm{N}=106)\end{array}$ & $\begin{array}{l}\text { Placebo } \\
\text { Group } \\
(\mathrm{N}=106)\end{array}$ & P Value* \\
\hline \multicolumn{4}{|l|}{ Oral mucositis of WHO grade 3 or 4} \\
\hline Incidence — no. of patients (\%)† & $67(63)$ & $104(98)$ & $<0.001$ \\
\hline \multicolumn{4}{|l|}{ Duration - days } \\
\hline All patients & & & $<0.001$ \\
\hline Median & 3.0 & 9.0 & \\
\hline Range & $0-22$ & $0-27$ & \\
\hline $\begin{array}{c}\text { Patients with oral mucositis of } \\
\text { WHO grade } 3 \text { or } 4\end{array}$ & & & $<0.001$ \\
\hline Median & 6.0 & 9.0 & \\
\hline Range & $1-22$ & $1-27$ & \\
\hline \multicolumn{4}{|l|}{ Patient-reported outcomes (AUC) $\%$} \\
\hline Score for soreness of mouth and throat & & & $<0.001$ \\
\hline Median & 29.0 & 46.8 & \\
\hline Range & 0-98 & $0-110$ & \\
\hline Swallowing-limitation score & & & $<0.001$ \\
\hline Median & 22.5 & 38.3 & \\
\hline Range & 0-104 & $0-104$ & \\
\hline \multicolumn{4}{|l|}{$\begin{array}{c}\text { Functional Assessment of Cancer } \\
\text { Therapy general score }\end{array}$} \\
\hline \multicolumn{4}{|l|}{ Physical well-being domain } \\
\hline Median & 736.6 & 712.1 & 0.003 \\
\hline Range & $176-1033$ & $176-1014$ & \\
\hline \multicolumn{4}{|l|}{ Functional well-being domain } \\
\hline Median & 546.1 & 542.5 & 0.036 \\
\hline Range & $93-985$ & $93-1043$ & \\
\hline
\end{tabular}

* P values were calculated with the use of the generalized Cochran-MantelHaenszel test stratified according to study center.

$\uparrow P<0.001$ for the two-part statistical model, which simultaneously considered the incidence of oral mucositis of WHO grade 3 or 4 and the duration of oral mucositis of WHO grade 3 or 4 among patients with this adverse effect.

$\downarrow$ AUC denotes area under the curve of outcome plotted against time. A Likert scale was used. For Functional Assessment of Cancer Therapy general score, higher scores indicate a more favorable response (range of possible scores, 0 to 4 ), whereas higher scores for soreness of the mouth and throat and swallowing limitations indicate a worse response (range of possible scores, 0 to 4 ). 


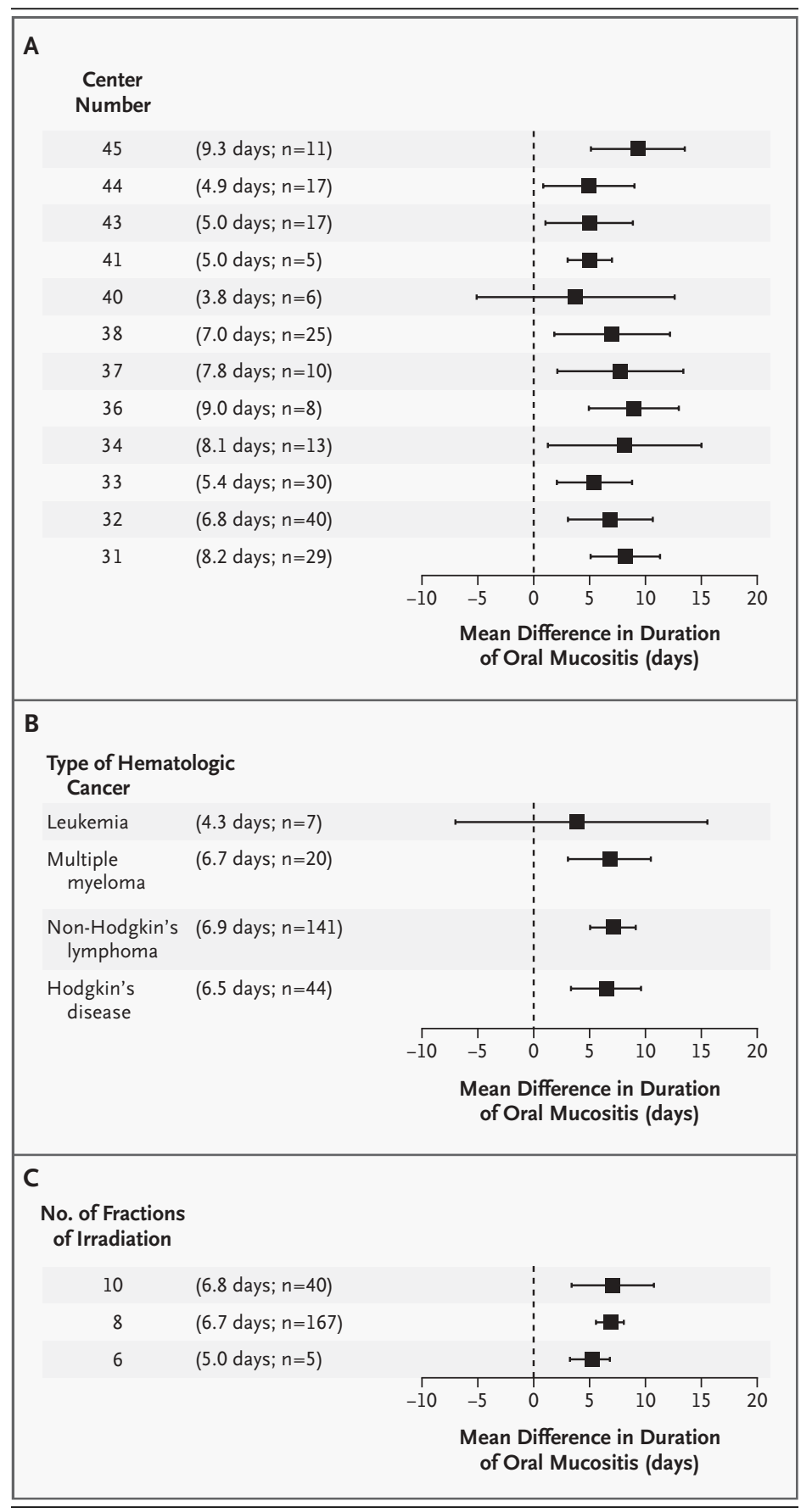

group (97 percent) and 102 patients in the placebo group ( 96 percent).

\section{EFFICACY}

Oral mucositis of WHO grade 3 or 4 developed in 67 of 106 patients in the palifermin group (63 percent) and 104 of 106 patients in the placebo group (98 percent, $\mathrm{P}<0.001$ ) (Table 2). The median dura-
Figure 1. Mean Difference between the Placebo Group and the Palifermin Group in the Duration of Mucositis of WHO Grade 3 or 4, According to Center (Panel A), Type of Hematologic Cancer (Panel B), and Number of Fractions of Total-Body Irradiation (Panel C).

Values in parentheses are the mean differences in duration between the placebo group and the palifermin group (also indicated by the squares) and the numbers of patients. Horizontal bars represent 95 percent confidence intervals. The mean difference could not be calculated for center 39 , which enrolled only one patient, who received palifermin; centers 35,42 , and 46 did not enroll any patients. The lower limit of the 95 percent confidence interval for the difference in duration exceeded 0 days for all centers except center 40 (six patients [four in the palifermin group and two in the placebo group]), for all hematologic cancers except leukemia (seven patients [two and five, respectively]), and for all three irradiation subgroups.

tion of oral mucositis of grade 3 or 4 among patients with this adverse effect was 6.0 days (range, 1 to 22 ) in the palifermin group and 9.0 days (range, 1 to 27$)$ in the placebo group $(\mathrm{P}<0.001)$ (Table 2$)$. The median duration of oral mucositis of WHO grade 3 or 4 among all patients was 3.0 days (range, 0 to 22 ) in the palifermin group and 9.0 days (range, 0 to 27 ) in the placebo group $(\mathrm{P}<0.001)$ (Table 2$)$. This result - the primary end point - was reproducible when the analysis was performed according to center, type of hematologic cancer, or number of fractions of irradiation (Fig. 1). A two-part statistical model, ${ }^{23}$ which simultaneously considered the incidence of oral mucositis of WHO grade 3 or 4 and the duration of oral mucositis of WHO grade 3 or 4 among patients with this adverse effect, showed significant improvements in the palifermin group, as compared with the placebo group $(\mathrm{P}<0.001)$ (Table 2).

The incidence of oral mucositis of WHO grade 4 (Fig. 2) was significantly lower among palifermin recipients than among placebo recipients (20 percent vs. 62 percent, $\mathrm{P}<0.001$ ), and the median duration of grade 4 oral mucositis was significantly shorter among the 21 palifermin recipients than among the 66 placebo recipients with this adverse effect (2.0 days [range, 1 to 9] vs. 6.0 days [range, 1 to 37 ], $\mathrm{P}=0.004$ ).

Approximately 3 percent of assessments for oral mucositis were missing. In a sensitivity analysis that imputed missing oral-mucositis assessments by assigning the worst possible score for palifermin and the best possible score for placebo, the median du- 


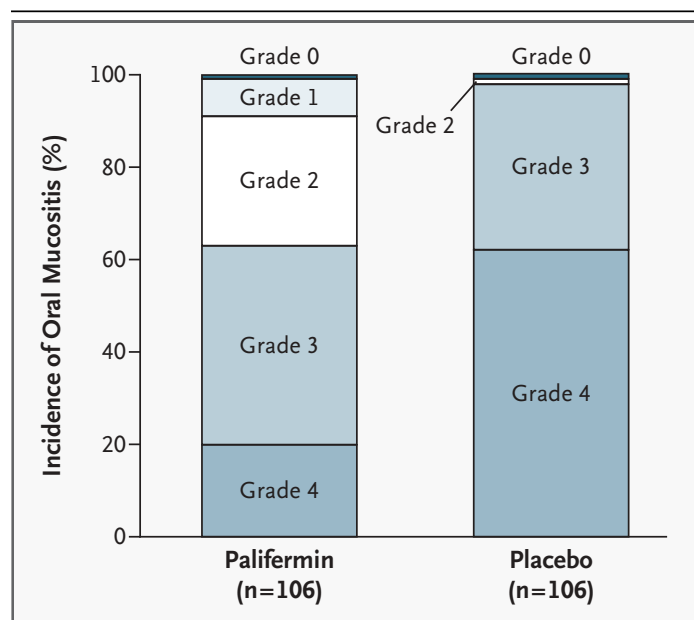

Figure 2. Incidence of Oral Mucositis in the Palifermin and Placebo Groups.

The severity of oral mucositis, as measured by the WHO assessment scale, is classified as follows: no oral mucositis (grade 0 ); soreness or erythema (grade 1); erythema and ulcers (grade 2); extensive erythema, ulcers, and inability to swallow solid food (grade 3 ); and mucositis that prevents any form of alimentation, including swallowing liquids (grade 4). No patient in the placebo group had grade 1 oral mucositis. The incidence of oral mucositis of WHO grade 3 or 4 was significantly lower in the palifermin group than in the placebo group $(P<0.001)$, as was the incidence of grade 4 oral mucositis $(P<0.001)$ and the incidence of oral mucositis of grade 2 or higher $(P<0.01)$.

ration of oral mucositis of grade 3 or 4 was still significantly shorter in the palifermin group than in the placebo group ( 3.0 days [range, 0 to 20] vs. 8.0 days [range, 0 to 27], $\mathrm{P}<0.001$ ).

Similar results were seen for other measurements of oral mucositis among all patients, including the median duration of oral mucositis of WHO grade 2 or higher ( 8.0 days [range, 0 to 28 ] in the palifermin group and 14.3 days [range, 0 to 37] in the placebo group, $\mathrm{P}<0.001$ ), the median duration of oral mucositis of RTOG grade 3 or $4(0.0$ days [range, 0 to 24] and 6.0 days [range, 0 to 54], $\mathrm{P}<0.001)$, and the median duration of lesions of WCCNR grade 2 or 3 (1.0 day [range, 0 to 36] and 7.0 days [range, 0 to 56 ], $\mathrm{P}<0.001$ ).

In agreement with the measurement of oral mucositis, the median scores for the soreness of the mouth and throat, measured by means of a Likert (categorical) scale, were significantly lower in the palifermin group than the placebo group (AUC, 29.0 [range, 0 to 98 ] vs. 46.8 [range, 0 to 110 ]; dif-

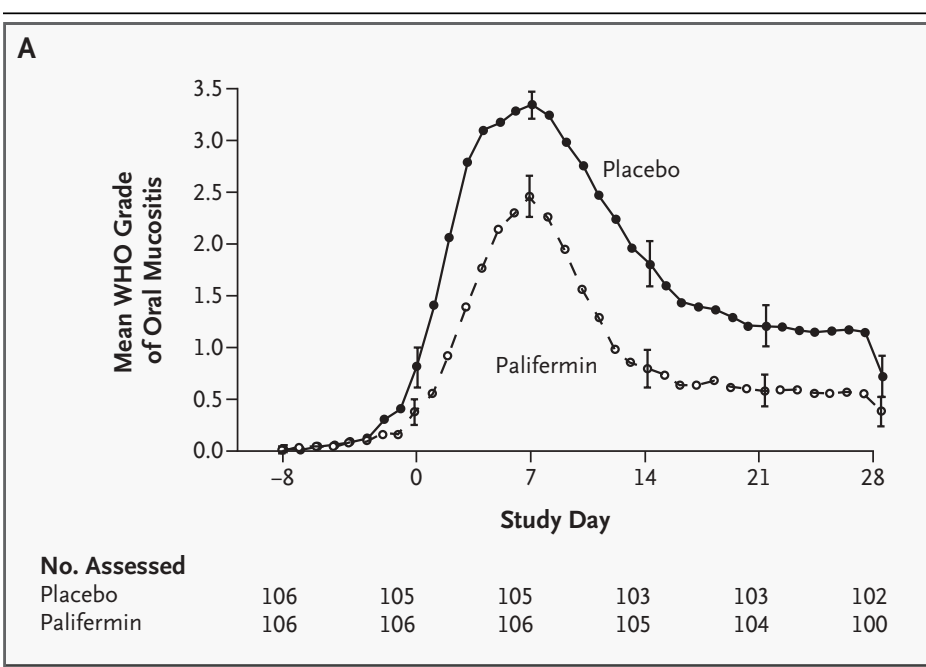

B

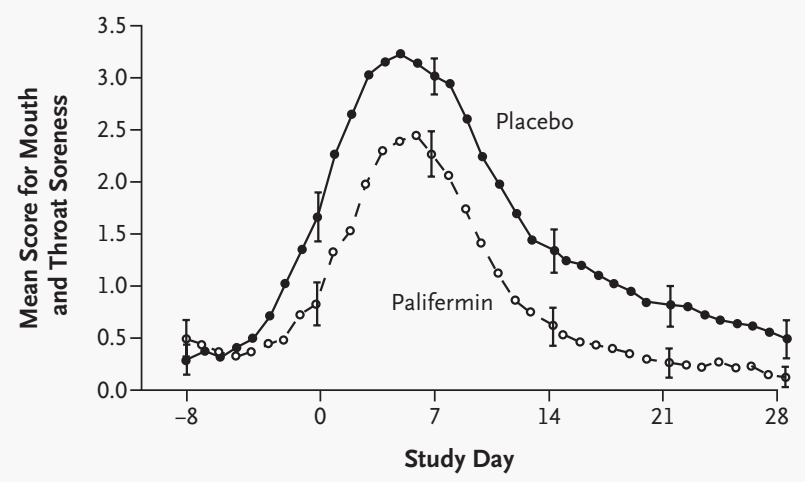

No. Assessed

Placebo

Palifermin

$\begin{array}{llllll}106 & 103 & 101 & 99 & 96 & 79 \\ 105 & 105 & 103 & 99 & 97 & 85\end{array}$

Figure 3. Mean WHO Grade of Oral Mucositis (Panel A) and Scores for Soreness of the Mouth and Throat (Panel B) during the Study.

The WHO grades were determined by members of the evaluation team, and the scores for soreness of the mouth and throat were determined by the patients. For days -8 (before stem-cell transplantation), 0, 7, 14, 21, and 28, vertical lines represent 95 percent confidence intervals. Mouth soreness was graded on a four-point scale, with higher scores indicating greater soreness.

ference between groups, 38 percent; $\mathrm{P}<0.001)$ (Table 2). By comparing daily mean scores for soreness of the mouth and throat with the WHO grade of oral mucositis, we found that the changes in the patientreported scores for soreness of the mouth and throat preceded the observer-reported changes in the grade of oral mucositis (Fig. 3). Similar results were observed for the incidence and duration of swallowing limitations (Table 2) and other patientreported sequelae related to soreness of the mouth and throat (e.g., ability to drink, eat, talk, and sleep) (data not shown). Palifermin recipients had signif- 


\begin{tabular}{|lcc|}
\hline \multicolumn{3}{|c|}{ Table 3. Adverse Events Occurring with an Incidence That Was at Least } \\
$\mathbf{5}$ Percentage Points Higher in the Palifermin Group Than in the Placebo Group.
\end{tabular}

icantly higher scores for the physical and functional well-being domains of the Functional Assessment of Cancer Therapy general questionnaire than did placebo recipients, also indicating greater improvement (Table 2).

Palifermin recipients used less parenteral or transdermal opioid analgesics for mucositis than did placebo recipients, as measured by the median cumulative dose administered (212 mg of morphine equivalents [range, 0 to 9418 ] vs. $535 \mathrm{mg}$ of morphine equivalents [range, 0 to 9418], $\mathrm{P}<0.001$ ) and the median duration of administration (7.0 days [range, 0 to 28] vs. 11.0 days [range, 0 to 32], $\mathrm{P}<0.001$ ). Palifermin recipients had a lower incidence of febrile neutropenia than did placebo recipients (75 percent vs. 92 percent, $\mathrm{P}<0.001)$. Exploratory analysis revealed a trend toward a lower incidence of blood-borne infections in the palifermin group than in the placebo group (15 percent vs. 25 percent). The incidence of the use of total parenteral nutrition during the study was also lower among palifermin recipients than among placebo recipients ( 31 percent vs. 55 percent, $\mathrm{P}<0.001$ ).

\section{SAFETY}

The incidence, frequency, and severity of adverse events were similar in the two groups, and most were attributable to the underlying cancer, cytotoxic chemotherapy, or total-body irradiation. Those that occurred with an incidence that was at least 5 percentage points higher in the palifermin group than in the placebo group are listed in Table 3. Most of these adverse events were consistent with the pharmacologic action of palifermin on skin and oral epithelium (e.g., rash, pruritus, erythema, paresthesia, mouth and tongue disorders, and taste alteration). All these events were mild to moderate in severity, transient (occurring approximately three days after the third dose of palifermin and lasting approximately three days), and not a cause for the discontinuation of study drug.

Serious adverse events considered to be related to treatment occurred in one palifermin recipient (rash) and one placebo recipient (hypotension). Two patients died during the study or within 30 days after the last dose of palifermin or placebo: one patient in the palifermin group died of veno-occlusive liver disease, and one patient in the placebo group died of sepsis. Neither death was considered to be related to treatment.

Data collection on hematologic recovery after transplantation was not prospectively specified; however, by day 12 after transplantation, most palifermin recipients ( 90 percent) and placebo recipients ( 93 percent) had absolute neutrophil counts of at least 500 per cubic millimeter.

Transient, asymptomatic increases in serum amylase (primarily of salivary origin) and lipase concentrations were observed in both groups, with the peak value for amylase occurring on the last day of irradiation and the peak value for lipase occurring after the third dose of study drug. The increases were higher in the palifermin group (median maximal increases from baseline, $166.5 \mathrm{U}$ of amylase per liter and $17.5 \mathrm{U}$ of lipase per liter) than the placebo group (92.0 and 12.5 U per liter, respectively). No additional increase was observed for either enzyme, and concentrations returned to near-baseline values by the day of transplantation.

No clinically significant differences were observed between groups in other laboratory results. Increases from baseline in WHO toxicity grades of alanine aminotransferase, aspartate aminotransferase, bilirubin, alkaline phosphatase, and creatinine concentrations - primarily one-grade increases were observed in less than 35 percent of patients in each group. Antibodies against palifermin were not detectable in any patient.

As of November 2003, the median duration of 
follow-up was 12.6 months in the palifermin group and 12.0 months in the placebo group. The KaplanMeier estimate of the rate of progression-free survival at 12 months was 0.69 (95 percent confidence interval, 0.60 to 0.78 ) in the palifermin group and 0.73 (95 percent confidence interval, 0.64 to 0.82 ) in the placebo group. In follow-up studies to date, secondary cancers have been diagnosed in three patients: myelodysplastic syndrome in one patient in the palifermin group and one patient in the placebo group and acute lymphoblastic leukemia in one patient in the palifermin group.

\section{ISCUSSIO N}

This study provides evidence that palifermin can significantly reduce, in a clinically meaningful way, the duration and incidence of oral mucositis after intensive chemotherapy and radiotherapy and autologous hematopoietic stem-cell transplantation. Palifermin consistently decreased the incidence and duration of severe oral mucositis and its clinical sequelae independently of the measure used, the participating center, the type of underlying disease, and the number of radiation fractions used. The intensive training of study personnel on the assessment of oral mucositis enhanced the quality of the data, and the quantity of missing data was minimal (approximately 3 percent), increasing confidence in the results. In addition, observer-reported assessments concurred with patient-reported assessments of the soreness of the mouth and throat and related functions, including swallowing.

An important finding was that palifermin markedly reduced the incidence of oral mucositis of WHO grade 4 (20 percent, as compared with 62 percent in the placebo group; $\mathrm{P}<0.001$ ), the most debilitating form of oral mucositis, in which oral alimentation is impossible. It is consistent with these findings that the percentage of patients who received total parenteral nutrition, an intervention frequently required for severe oral mucositis, ${ }^{24,25}$ and the amount and duration of use of parenteral opioid analgesics were significantly lower among palifermin recipients than placebo recipients.

Our results are particularly compelling because of the severity and duration of oral mucositis typically seen in patients with hematologic cancers who undergo intensive treatment. Although antibiotics and topical palliative agents can help manage infections and pain resulting from mild-to-moderate oral mucositis, none of the growth factors and cytokines unrelated to keratinocyte growth factor tested to date have demonstrated a clear benefit in the setting of oral mucositis. ${ }^{7,26-30}$

Severe oral mucositis is associated with an increased risk of blood-borne bacterial infections, which can be life-threatening. ${ }^{31,32}$ Our exploratory analysis of blood-borne infections showed a lower incidence among patients receiving palifermin than among those receiving placebo. If confirmed by future investigations, the ability of palifermin therapy to reduce the breakdown of mucosal barriers could decrease the complications caused by systemic infection. In turn, reducing the complications of oral mucositis could decrease the use of parenteral or transdermal opioid analgesics for mucositis and parenteral nutrition and reduce the length of hospitalization.

Palifermin is a growth factor. Keratinocyte growth-factor receptor is not known to be expressed in hematologic cancers ${ }^{33}$; nevertheless, the growth of second tumors that express this receptor is theoretically possible. Evaluation of this risk requires long-term follow-up, which is ongoing. At 12 months, the progression-free survival rates for palifermin and placebo were nearly identical. In summary, $60 \mu \mathrm{g}$ of palifermin per kilogram per day was associated with reductions in the duration and severity of debilitating oral mucositis and related sequelae of high-dose myelosuppressive treatment with autologous hematopoietic stem-cell support.

Funded by Amgen.

Drs. Spielberger, Kewalramani, and Emmanouilides report having received consulting and lecture fees from Amgen; Drs. Stiff and Blazar, consulting fees, lecture fees, and grant support from Amgen; Dr. Bensinger, consulting fees and grant support from Amgen Dr. Shea, lecture fees and grant support from Amgen; and Dr. Noga, lecture fees from Amgen and Ortho Biotech. Dr. Elhardt and Ms. Chen are employees of Amgen and hold equity in Amgen.

We are indebted to Robert Heard, Ph.D., and Alessandra Cesano, M.D., for assistance with the conduct of the study; to Stephen Sonis, D.M.D., D.M.Sc., for advice regarding the assessment of oral mucositis; to M. Haim Erder, Ph.D., and Jon Ford, Ph.D., for evaluating patient-reported outcomes; to Alan Rong, Ph.D., and John Isitt, Ph.D., for assistance with statistical analysis; to Kathy Jelaca-Maxwell, R.N., for assistance with safety evaluations; to Don Smith, Ph.D., for editorial assistance; and to the patients who participated in this study, their families, and all the physicians, nurses, and study coordinators who cared for the patients. 
REFERENCES

1. McGuire DB, Altomonte V, Peterson DE Wingard JR, Jones RJ, Grochow LB. Patterns of mucositis and pain in patients receiving preparative chemotherapy and bone marrow transplantation. Oncol Nurs Forum 1993;20:1493-502.

2. Woo SB, Sonis ST, Monopoli MM, Sonis AL. A longitudinal study of oral ulcerative mucositis in bone marrow transplant recipients. Cancer 1993;72:1612-7.

3. Sonis ST. Mucositis as a biological process: a new hypothesis for the development of chemotherapy-induced stomatotoxicity. Oral Oncol 1998;34:39-43.

4. Bellm LA, Epstein JB, Rose-Ped A, Martin P, Fuchs HJ. Patient reports of complications of bone marrow transplantation. Sup port Care Cancer 2000;8:33-9.

5. Sonis ST, Oster G, Fuchs H, et al. Ora mucositis and the clinical and economic outcomes of hematopoietic stem-cell transplantation. J Clin Oncol 2001;19:2201-5.

6. Armstrong TS. Stomatitis in the bone marrow transplant patient: an overview and proposed oral care protocol. Cancer Nurs 1994;17:403-10.

7. Donnelly JP, Blijlevens NMA, Verhagen $\mathrm{CAH}$. Can anything be done about oral mucositis? Ann Oncol 2003;14:505-7.

8. Rubin JS, Osada H, Finch PW, Taylor WG, Rudikoff S, Aaronson SA. Purification and characterization of a newly identified growth factor specific for epithelial cells Proc Natl Acad Sci U S A 1989;86:802-6.

9. Farrell CL, Bready JV, Rex KL, et al. Keratinocyte growth factor protects mice from chemotherapy and radiation-induced gastrointestinal injury and mortality. Cancer Res 1998;58:933-9.

10. Farrell CL, Rex KL, Kaufman SA, et al. Effects of keratinocyte growth factor in the squamous epithelium of the upper aerodigestive tract of normal and irradiated mice. Int J Radiat Biol 1999;75:609-20.

11. Meropol NJ, Somer RA, Gutheil J, et al. Randomized phase I trial of recombinant human keratinocyte growth factor plus chemotherapy: potential role as mucosal protectant. J Clin Oncol 2003;21:1452-8

12. Blume KG, Forman SJ, O'Donnell MR, et al. Total body irradiation and high-dose etoposide: a new preparatory regimen for bone marrow transplantation in patient with advanced hematologic malignancies. Blood 1987;69:1015-20.

13. Horning SJ, Negrin RS, Chao NJ, Long
GD, Hoppe RT, Blume KG. Fractionated total-body irradiation, etoposide, and cyclophosphamide plus autografting in Hodgkin's disease and non-Hodgkin's lymphoma. J Clin Oncol 1994;12:2552-8.

14. Snyder DS, Chao NJ, Amylon MD, et al. Fractionated total body irradiation and high-dose etoposide as a preparatory regimen for bone marrow transplantation fo 99 patients with acute leukemia in first complete remission. Blood 1993;82:2920-8.

15. Stiff PJ, Dahlberg S, Forman SJ, et al. Autologous bone marrow transplantation for patients with relapsed or refractory diffuse aggressive non-Hodgkin's lymphoma: value of augmented preparative regimens - a Southwest Oncology Group trial. J Clin Oncol 1998;16:48-55.

16. Spielberger, RT, Stiff P, Emmanouilide $\mathrm{C}$, et al. Efficacy of recombinant human keratinocyte growth factor (rHuKGF) in reducing mucositis in patients with hematologic malignancies undergoing autologous peripheral blood progenitor cell transplantation (auto-PBPCT) after radiation-based conditioning: results of a phase 2 trial. Proc Am Soc Clin Oncol 2001;20:7a. abstract.

17. Miller $A B$, Hoogstraten $B$, Staquet $M$, Winkler A. Reporting results of cancer treatment. Cancer 1981;47:207-14.

18. National Cancer Institute common toxicity criteria, version 2.0. April 30, 1999. (Ac cessed November 19, 2004, at http://ctep. info.nih.gov.)

19. Assessing stomatitis: refinement of the Western Consortium for Cancer Nursing Research (WCCNR) stomatitis staging system. Can Oncol Nurs J 1998;8:160-5.

20. Cella DF, Tulsky DS, Gray G, et al. The Functional Assessment of Cancer Therapy scale: development and validation of the general measure. J Clin Oncol 1993;11:570-9.

21. Landis JR, Heyman ER, Koch GG. Average partial association in three-way contingency tables: a review and discussion of alternative tests. Int Stat Rev 1978;46:237-54. 22. Kalbfleisch JD, Prentice PL. The statistical analysis of failure time data. New York John Wiley, 1980.

23. Lachenbruch PA. Analysis of data with clumping at zero. Biometrische Z 1976;18: 351-6.

24. Kolbinson DA, Schubert MM, Fluornoy $\mathrm{N}$, Truelove EL. Early oral changes following bone marrow transplantation. Oral Surg Oral Med Oral Pathol 1988;66:130-8.
25. Zerbe MB, Parkerson SG, Ortlieb ML Spitzer T. Relationships between oral mucositis and treatment variables in bone mar row transplant patients. Cancer Nurs 1992; 15:196-205.

26. Foncuberta MC, Cagnoni PJ, Brandts $\mathrm{CH}$, et al. Topical transforming growth factor- $\beta 3$ in the prevention or alleviation of chemotherapy-induced oral mucositis in patients with lymphomas or solid tumors. J Immunother 2001;24:384-8.

27. Stiff P. Mucositis associated with stem cell transplantation: current status and innovative approaches to management. Bone Marrow Transplant 2001;27:Suppl 2:S3-S11. 28. Antin JH, Lee SJ, Neuberg D, et al. A phase I/II double-blind, placebo-controlled study of recombinant human interleukin-11 for mucositis and acute GVHD prevention in allogeneic stem cell transplantation. Bone Marrow Transplant 2002;29:373-7.

29. Dazzi C, Cariello A, Giovanis P, et al. Prophylaxis with GM-CSF mouthwashes does not reduce frequency and duration of severe oral mucositis in patients with solid tumors undergoing high-dose chemotherapy with autologous peripheral blood stem cell transplantation rescue: a double blind, randomized, placebo-controlled study. Ann Oncol 2003;14:559-63.

30. Filicko J, Lazarus HM, Flomenberg N. Mucosal injury in patients undergoing hematopoietic progenitor cell transplantation: new approaches to prophylaxis and treatment. Bone Marrow Transplant 2003;31:110.

31. Rapoport AP, Miller Watelet LF, Linder T, et al. Analysis of factors that correlate with mucositis in recipients of autologous and allogeneic stem-cell transplants. J Clin Oncol 1999;17:2446-53.

32. Sezer O, Eucker J, Metzner B, et al. Mucositis is associated with increased rate of documented infections and treatment-related mortality after high-dose therapy with autologous peripheral stem-cell transplantation. Proc Am Soc Clin Oncol 2000;19: 56a. abstract.

33. Moroni E, Dell'Era, P, Rusnati M, Presta M. Fibroblast growth factors and their receptors in hematopoiesis and hematological tumors. J Hematother Stem Cell Res 2002;11:19-32

Copyright (c) 2004 Massachusetts Medical Society. 